\title{
Suppression of circ_0008932 inhibits tumor growth and metastasis in osteosarcoma by targeting miR-145-5p
}

\author{
CHENGGANG CAO $^{1}$ and XIAOLEI $\mathrm{SHU}^{2}$
}

\author{
${ }^{1}$ Department of Orthopedics, Chongqing Traditional Chinese Medicine Hospital/Chongqing First People's Hospital, \\ Chongqing 400011; ${ }^{2}$ Department of Radiation Oncology, Chongqing Key Laboratory of Translational Research for Cancer \\ Metastasis and Individualized Treatment, Chongqing University Cancer Hospital, Chongqing 400030, P.R. China
}

Received December 26, 2020; Accepted June 17, 2021

DOI: $10.3892 /$ etm.2021.10540

\begin{abstract}
Osteosarcoma (OS) is a common type of primary malignant tumor. Although the pathogenesis of OS has been extensively studied, the underlying molecular mechanisms have remained to be fully elucidated. Accumulating evidence has revealed that dysregulation of various circular RNAs (circRNAs) is associated with tumorigenesis and recent studies have indicated that circRNA circ_0008932 is aberrantly expressed in tumors. In the present study, the expression and detailed function of circ_0008932 in OS were elucidated. The levels of circ_0008932 in OS samples and cell lines were examined using reverse transcription-quantitative PCR. A cell model with circ_0008932 knockdown was generated using specific small interfering RNA (si-circ_0008932). Cell viability was determined by a Cell Counting Kit-8 assay, the cell migratory/invasive capacity was evaluated using Transwell assays and cell apoptosis was assessed by flow cytometry. The results suggested that circ_0008932 was upregulated in most primary OS tumors, suggesting that circ_0008932 is associated with the development of OS. In the in vitro assays, si-circ_0008932 inhibited the proliferation, migration and invasion of OS cells, while apoptosis was promoted. A luciferase reporter assay revealed that circ_0008932 may downregulate microRNA (miR)-145-5p through direct binding. Furthermore, the expression of miR-145-5p was negatively correlated with circ_0008932 levels in OS specimens. In addition, further functional studies indicated that miR-145-5p inhibitors eliminated the effects caused by si-circ_0008932 in OS cells. In comparison, the changes in the biological behavior of OS cells transfected with si-circ_0008932 were enhanced by miR-145-5p. In summary, circ_0008932 may be a novel
\end{abstract}

Correspondence to: Dr Xiaolei Shu, Department of Radiation Oncology, Chongqing Key Laboratory of Translational Research for Cancer Metastasis and Individualized Treatment, Chongqing University Cancer Hospital, 181 Huanyu Road, Chongqing 400030, P.R. China

E-mail: shux1881@163.com

Key words: non-coding RNA, osteosarcoma, oncogenesis oncogenic factor during the progression and development of OS by targeting miR-145-5p; more importantly, circ_0008932 may be a potential therapeutic target for OS.

\section{Introduction}

Osteosarcoma (OS) is one of the most common primary malignancies and it mainly arises in the metaphyses of long bones in adolescents and young adults (1). OS is associated with abnormal cell differentiation caused by genetic and epigenetic alterations (2). Although treatment methods, including chemoand radiotherapy and surgery, have been developed and are in use, the prognosis of patients with recurrent and metastatic OS remains poor with a five-year survival rate of $50-60 \%$ (3). It has been indicated that multiple oncogenes and tumor suppressors are involved in the occurrence and development of OS (4). However, the detailed molecular mechanisms of OS and processes associated with its progression, including migration and invasion, have remained to be fully elucidated.

Circular RNAs (circRNAs) are a group of non-coding RNAs, which are widely expressed in numerous species. They are characterized as having covalent and closed-loop structures (5). CircRNAs are considered key epigenetic regulators in the pathogenesis of various diseases and they may have essential roles in tumor genesis and development (6). For instance, circ-0000658 was downregulated in OS tissues and cells, which inhibited the cell cycle, proliferation, invasion and migration of cells through the microRNA (miRNA/miR)-1227/IRF2 axis (7). Silencing of circ_0032462 inhibited OS cell proliferation, migration and invasion by upregulating kinesin family member 3B (8). Circ_0008932 was significantly upregulated in esophageal squamous cell carcinoma (ESCC) and knockdown of circ_0008932 reduced the viability, colony formation ability and invasion of ESCC cells through targeting the miR-182/Myc axis (9). Circ_0008932 suppressed vascular smooth muscle cell (VSMC) migration, proliferation and differentiation by binding to miR-145 $(10,11)$. However, the roles of circ_0008932 in OS have not been previously studied.

In the present study, it was revealed that circ_0008932 was upregulated in osteosarcoma tissues compared to corresponding noncancerous samples and circ_0008932 upregulation was also associated with metastasis in patients with OS. The 
survival analysis demonstrated that high circ_0008932 expression in OS tissues was associated with an unfavorable overall survival prognosis. Suppression of circ_0008932 significantly inhibited the proliferation, invasion and migration of OS cells, while cell apoptosis was promoted. Furthermore, the present data indicated that circ_0008932 directly downregulated miR-145-5p expression, which was negatively correlated with circ_0008932 expression in OS tissues.

\section{Materials and methods}

OS tissues and cell lines. A total of 50 OS tissues and matched non-tumor tissues were collected from all patients ( 27 males and 23 females; mean age, $6.36 \pm 2.18$ years) during surgery at the Department of Radiation Oncology, Chongqing University Cancer Hospital (Chonqing, China), where metastasis was detected in 29 cases. Metastatic tissues were not analyzed in this study. Written informed consent was provided by all patients. The protocol was approved by the Ethics Committee of Chongqing University Cancer Hospital (Chongqing, China). Upon collection, tissues were immediately frozen using liquid nitrogen and stored at $-80^{\circ} \mathrm{C}$ prior to RNA isolation. Cell lines, including NHOst, HOS, SOSP-9607, SaOS-2 and U2OS, were purchased from the Cell Bank of the Chinese Academy of Sciences and cultured using Dulbecco's modified Eagle's medium (DMEM; Invitrogen; Thermo Fisher Scientific, Inc.) supplemented with $10 \%$ fetal bovine serum (FBS; HyClone; Cytiva).

Cell transfection. Small interfering (si)RNA against circ_0008932 (si-circ_0008932; 5'-AACCACUGCCUGCCU CAAUCU-3') and a scrambled control siRNA used as a negative control (si-NC; 5'-UUACGUUACAAAUCCGAUGUA $\left.-3^{\prime}\right)$ were purchased from Shanghai GenePharma Co., Ltd. miR-145-5p mimics (5'-GUCCAGUUUUCCCAGGAAUCC CU-3') and the respective control (miR-NC; 5'-AAUUGAAGU UCCCAGGAAUCCCU-3'), as well as miR-145-5p inhibitor (5'-AGGGAUUCCUGGGAAAACUGGAC-3') and its control (in-NC; 5'-CAGUACUUUUGUGUAGUACAAA-3') were synthesized by Shanghai GenePharma Co., Ltd. siRNAs, miR mimics, miR inhibitors and their NC oligonucleotides $(50 \mathrm{nM})$ were transfected using Lipofectamine ${ }^{\circledR} 2000$ reagent (Invitrogen; Thermo Fisher Scientific, Inc.) according to the manufacturer's protocol.

$R N A$ extraction and reverse transcription-quantitative ( $R T-q)$ $P C R$. Total RNA was extracted from cells and tissues using the TRIzol ${ }^{\circledR}$ reagent (Invitrogen; Thermo Fisher Scientific, Inc.) according to the manufacturer's protocol. PrimeScript ${ }^{\mathrm{TM}}$ RT kit (cat. no. RR036B; Takara Biotechnology Co., Ltd.) was used for reverse transcription, the sample was incubated at room temperature for $30 \mathrm{~min}$. After that, 1 cycle of PCR was performed at $42^{\circ} \mathrm{C}$ for $45 \mathrm{~min}, 99^{\circ} \mathrm{C}$ for $5 \mathrm{~min}$ and $5^{\circ} \mathrm{C}$ for $5 \mathrm{~min}$ in a PCR cycler. qPCR analyses of circ_0008932 and miR-145-5p were performed using TB Green ${ }^{\circledR}$ Premix Ex Taq $^{\text {TM }}$ (cat. no. RR420A; Takara Bio, Inc.) in an ABI Prism 7700 system (Applied Biosystems; Thermo Fisher Scientific, Inc.). The thermocycling conditions contained initial denaturation at $95^{\circ} \mathrm{C}$ for $10 \mathrm{~min}$, followed by 40 cycles of $95^{\circ} \mathrm{C}$ for $10 \mathrm{sec}, 60^{\circ} \mathrm{C}$ for $30 \mathrm{sec}$ and $72^{\circ} \mathrm{C}$ for $10 \mathrm{sec}$ and final extension at $72^{\circ} \mathrm{C}$ for another $5 \mathrm{~min}$. Primers for U6 and miR-145-5p were purchased from GeneCopoeia, Inc. The primer sequences were as follows: circ_0008932 forward, 5'-TTTGGTCCCCTT CAACCAGCTG-3' and reverse, 5'-TAAACCAAGGTAAAA TGGTCGA-3'; miR-145-5p forward, 5'-GTCCAGTTTTCC CAGGAATCC-3' and reverse, 5'-TCGGCAGGGTCCAGT TTTCCCA-3'; GAPDH forward, 5'-CATGGCCTTCCGTGT TCCTA-3' and reverse, 5'-TACTTGGCAGGTTTCTCCAGG -3 ' and U6 forward, 5'-GCTTCGGCAGCACATATACTA AAAT-3' and reverse, 5'-CGCTTCACGAATTTGCGTGTC AT-3'. The expression of circ_0008932 was normalized to that of GAPDH and the levels of miR-145-5p were normalized to those of U6. The expression was calculated using the $2^{-\Delta \Delta \mathrm{Cq}}$ method (12). Patients were divided into circ_0008932 high- or low-expression groups according to the mean circ_0008932 expression value. All experiments were performed in triplicate.

Cell proliferation assay. SaOS-2 and U2OS cells were seeded in 96-well plates at a density of $1 \times 10^{3}$ cells per well. The cells were transfected with si-NC or si-circ_0008932 or co-transfected with miR-145-5p mimics/inhibitors and si-circ_0008932. Cell viability was evaluated for 4 consecutive days by using a Cell Counting Kit (CCK)-8 assay (Dojindo Molecular Technologies, Inc.). A total of $10 \mu \mathrm{l} \mathrm{CCK-8} \mathrm{reagent}$ was added to each well and the cells were incubated at $37^{\circ} \mathrm{C}$ for another $2 \mathrm{~h}$. The absorbance at $450 \mathrm{~nm}$ was measured using a microplate reader (Bio-Rad Laboratories). All experiments were independent and performed in triplicate.

Cell migration and invasion assays. For the cell invasion and migration assays, $1.0 \times 10^{5} \mathrm{SaOS}-2$ or U2OS cells were collected after transfection and used for each experiment. Cell migration and invasion were determined using Transwell assays. In brief, the suspension of cells containing no FBS was seeded into the upper chamber of the Transwell insert with a pore size of $8 \mu \mathrm{m}$ (BD Biosciences). In addition, for the cell invasion assay, cells were seeded onto the upper chambers pre-coated with Matrigel $^{\circledR}$ (Sigma-Aldrich; Merck KGaA). DMEM with $10 \%$ FBS was added into lower chamber. Cells were incubated at $37^{\circ} \mathrm{C}$ for $12 \mathrm{~h}$ and those that migrated or invaded through the membrane were fixed with $4 \%$ polyoxymethylene at room temperature for $10 \mathrm{~min}$, stained with $0.2 \%$ crystal violet at room temperature for $10 \mathrm{~min}$ and then counted under an IX83 inverted fluorescent microscope (magnification, x200; Olympus Corporation).

Luciferase assay. Bioinformatics analysis was performed using Targetscan (release 5.2; www.targetscan.org) and miRNA.org (version 10.0; www.microrna.org/microrna). To investigate the interaction between circ_0008932 and miR-145-5p, wild-type fragment of the 3'-untranslated region (UTR) on circ_0008932 with complementary binding sites of miR-145-5p were obtained from Shanghai GenePharma Co., Ltd. A circ_0008932 3'UTR-mutant (MUT) vector containing the mutant binding site of miR-145-5p was produced using a QuikChange Multi Site-Directed Mutagenesis kit (Stratagene; Agilent Technologies, Inc.) using the restriction digestion sites of SpeI and HindIII (Promega Corporation). The pmirGLO Dual-Luciferase plasmids (Promega Corporation) were used to construct the 

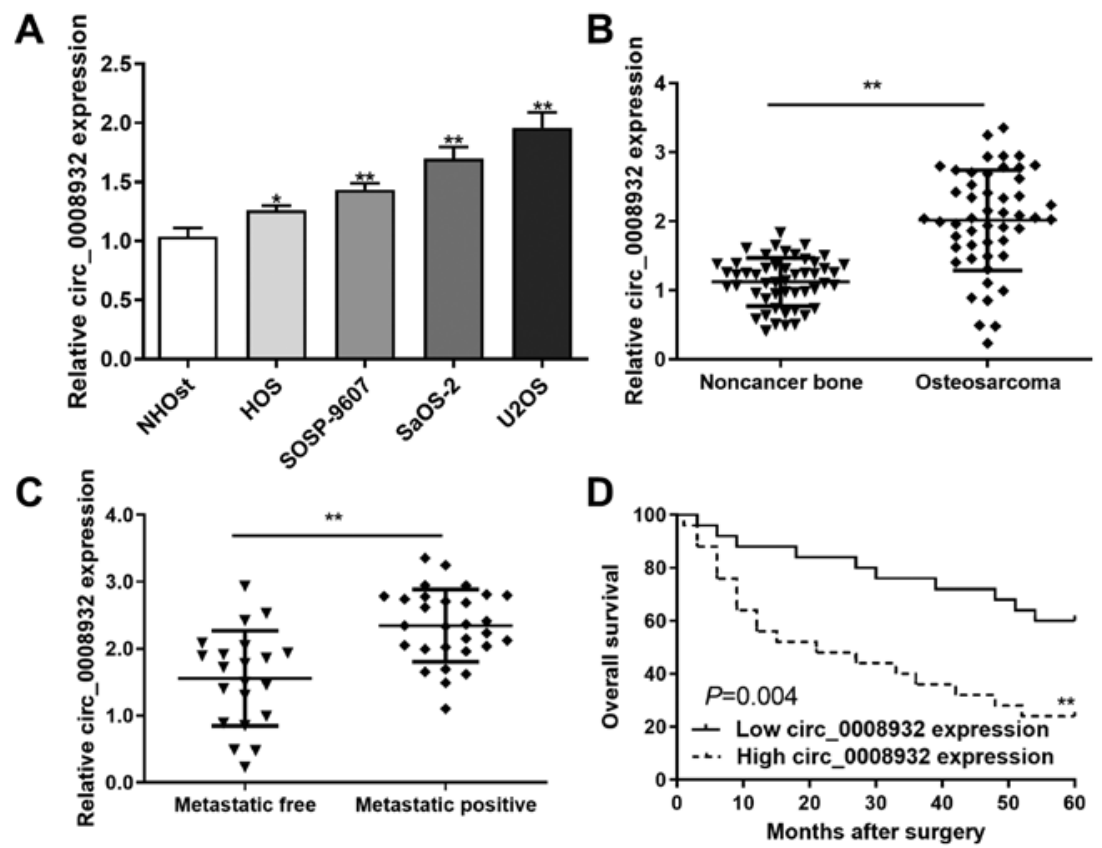

Figure 1.Circ_0008932 was upregulated in OS tissues and cells. (A) The expression of circ_0008932 in normal NHOst and four OS cell lines (HOS, SOSP-9607, SaOS-2 and U2OS) was examined using reverse transcription-quantitative PCR. (B) The expression of circ_0008932 in 50 OS and matched normal tissues was determined. (C) Levels of circ_0008932 in primary OS cases with or without metastasis. (D) Kaplan-Meier curves for the overall survival in the low- and high-circ_0008932 expression groups. ${ }^{*} \mathrm{P}<0.05,{ }^{* *} \mathrm{P}<0.01$ vs. NHOst, low-circ_0008932 expression or as indicated. OS, osteosarcoma; circ, circular RNA; NHOst, normal osteoblast cells.

luciferase reporter vectors. Mutant circ_0008932 was used as a control. For circ_0008932 (https://circinteractome.nia.nih. gov/api/v2/circsearch?circular_rna_query=hsa_circ_0008932\&gene_ symbol_query=\&submit=circRNA+Search), Homo sapiens full open reading frame cDNA of circ_0008932 was selected. The products were amplified using primers with flanking SpeI and HindIII restriction digestion sites and the amplified DNA was then inserted into the pmirGLO Dual-Luciferase reporter vector (Promega Corporation). The 293T cells (The Cell Bank of Type Culture Collection of the Chinese Academy of Sciences) were cultured with serum-free DMEM at $37^{\circ} \mathrm{C}$ with $5 \%$ of $\mathrm{CO}_{2}$. Cells were transfected with $25 \mathrm{nM}$ wild-type or mutant pMIR-circ_0008932, as well as Renilla luciferase control vector (pRL-TK; Promega Corporation) using Lipofectamine ${ }^{\circledR} 2000$. Cells were co-transfected with $25 \mathrm{nM}$ miR-NC or miR-145-5p mimics/inhibitors, respectively. Luciferase activity was examined $48 \mathrm{~h}$ after transfection using the Dual-Luciferase reporter assay system (Promega Corporation) using an LB 960 Centro XS3 luminometer (Molecular Devices, LLC).

Flow cytometry. Flow cytometry was used to determine cell apoptosis. Transfected cells $\left(1 \times 10^{5}\right.$ per well) were assayed using the Annexin V-FITC/propidium iodide (PI) apoptosis detection kit (Beijing Solarbio Science \& Technology Co., Ltd.) according to the manufacturer's protocol. Cells were stained with annexin V-FITC and propidium iodide (PI). After a $10 \mathrm{~min}$ incubation at room temperature, the cells were analyzed using a BD FACSAria III cytometer (BD Biosciences) and interpreted by ModFit software (version 2.0; BD Biosciences).

Statistical analysis. Values are expressed as the mean \pm standard deviation and analyzed using SPSS 19.0 (IBM Corporation).
Statistical significance was examined using Student's t-test or one-way ANOVA. Tukey's test was conducted as a post-hoc test following ANOVA. Patient survival was assessed using the Kaplan-Meier method and the log-rank test was applied to determine significant differences between groups. Pearson's correlation analysis was used to evaluate the relationship between the expression levels of circ_0008932 and miR-145-5p. $\mathrm{P}<0.05$ was considered to indicate statistical significance.

\section{Results}

Circ_0008932 is upregulated in OS tissues and cells and high levels are associated with metastasis and prognosis. RT-qPCR was performed to detect the levels of circ_0008932 in OS tissues and cells. The expression of circ_0008932 was assessed in five cell lines. The results indicated that the expression of circ_0008932 was increased in the four OS cell lines (HOS, SOSP-9607, SaOS-2 and U2OS) compared with that in NHOst normal human osteoblast cells (Fig. 1A). Furthermore, the expression of circ_0008932 in OS and paired non-tumor tissues was also evaluated. The results indicated that circ_0008932 expression was significantly increased in OS samples (Fig. 1B). In addition, circ_0008932 expression was increased in primary tumors of patients with metastasis compared with those without metastasis (Fig. 1C). In addition, the overall survival of patients with OS with high circ_0008932 expression was poor compared with that of patients with low circ_0008932 expression ( $\mathrm{P}=0.004$; Fig. 1D).

Silencing of circ_0008932 inhibits the proliferation, invasion and migration of $O S$ cells, whereas cell apoptosis is enhanced. To determine whether the inhibition of circ_0008932 is able to suppress the proliferation, migration 
A

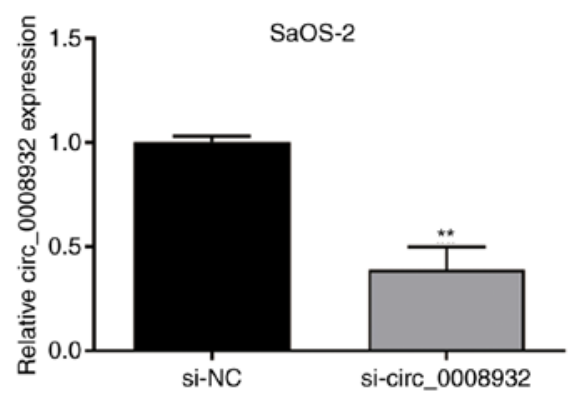

C

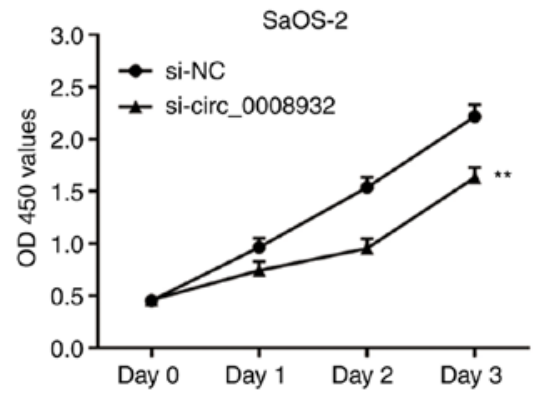

E

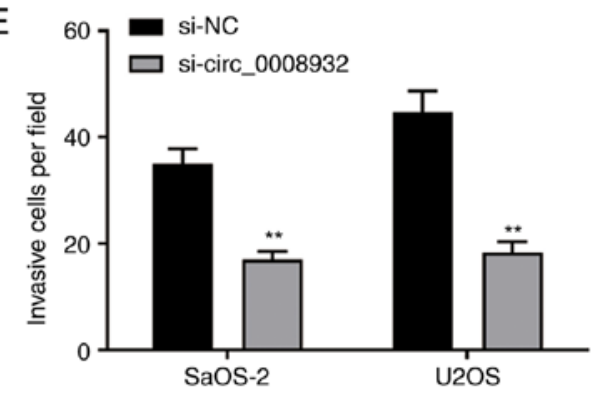

G

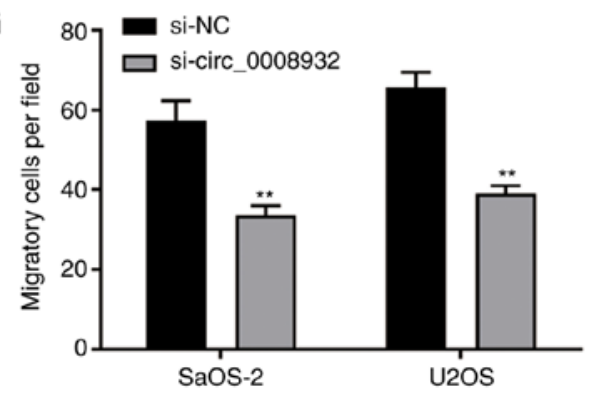

$\mathrm{B}$
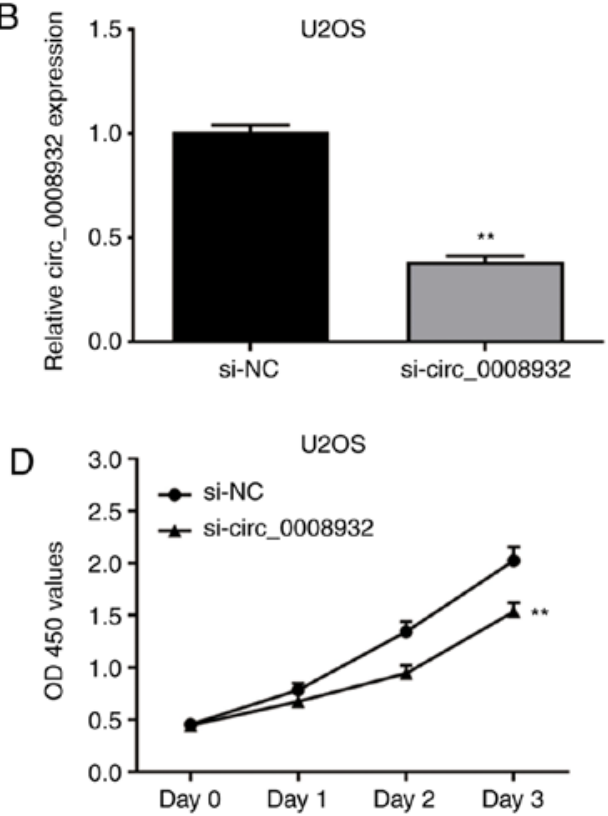

F

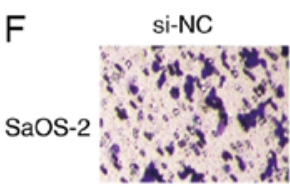

si-circ_0008932

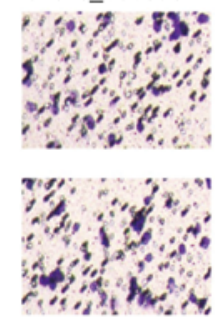

$\mathrm{H}$
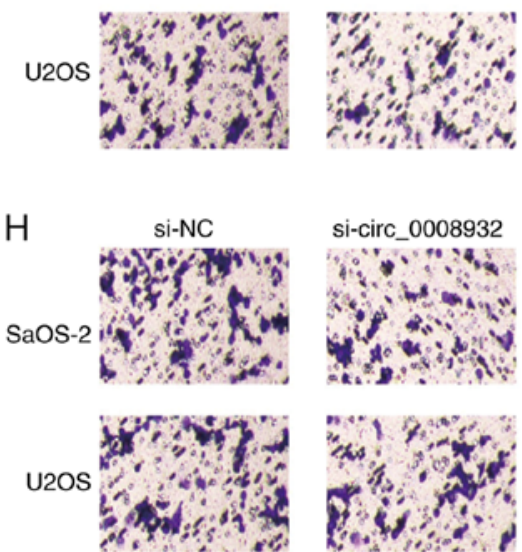

Figure 2. Silencing of circ_0008932 inhibits the proliferation, invasion and migration of osteosarcoma cells. (A and B) The expression of circ_0008932 in (A) SaOS-2 and (B) U2OS cells transfected with si-NC or si-circ_0008932 was detected by reverse transcription-quantitative PCR. (C and D) The proliferation of (C) SaOS-2 and (D) U2OS cells transfected with si-NC or si-circ_0008932 was evaluated using Cell Counting Kit-8 assays. (E-H) Transwell assays were used to assess invasion and migration of SaOS-2 and U2OS cells transfected with si-NC or si-circ_0008932. (E) Quantitative results and (F) representative images of cell invasion. (G) Quantitative results and (H) representative images of cell migration Magnification, $\mathrm{x} 200$. ${ }^{* * *} \mathrm{P}<0.01$ vs. si-NC. Circ, circular RNA; si, short interfering RNA; NC, negative control; OD, optical density.

and invasion of OS cells, SaOS-2 and U2OS cells were transfected with si-NC or si-circ_0008932 for $24 \mathrm{~h}$ and subsequently, cell proliferation, invasion and migration were examined. It was confirmed that the expression of circ_0008932 was significantly decreased in SaOS-2 and U2OS cells transfected with si-circ_0008932 (Fig. 2A and B). The results revealed that knockdown of circ_0008932 markedly suppressed cell proliferation (Fig. 2C and D), invasion (Fig. 2E and F) and migration (Fig. 2G and H), while cell apoptosis was promoted (Fig. 3). These data suggested that knockdown of circ_0008932 suppressed the proliferation/motility and promoted apoptosis of OS cells.
miR-145-5p is a direct target of circ_0008932. Bioinformatics analysis using Targetscan and miRNA.org indicated that circ_0008932 contains potential binding sites for miR-145-5p. To confirm whether miR-145-5p is a direct target of circ_0008932, luciferase vectors containing the wild-type or mutant circ_0008932 sequence were constructed (Fig. 4A). For the luciferase assays, wild-type or mutant plasmids were co-transfected into $293 \mathrm{~T}$ cells with miR-145-5p mimics or inhibitors. As presented in Fig. 4B, miR-145-5p mimics significantly reduced the luciferase activity of wild-type circ_0008932, while that of the mutant circ_0008932 was not affected. The results also suggested that knockdown of circ_0008932 significantly 

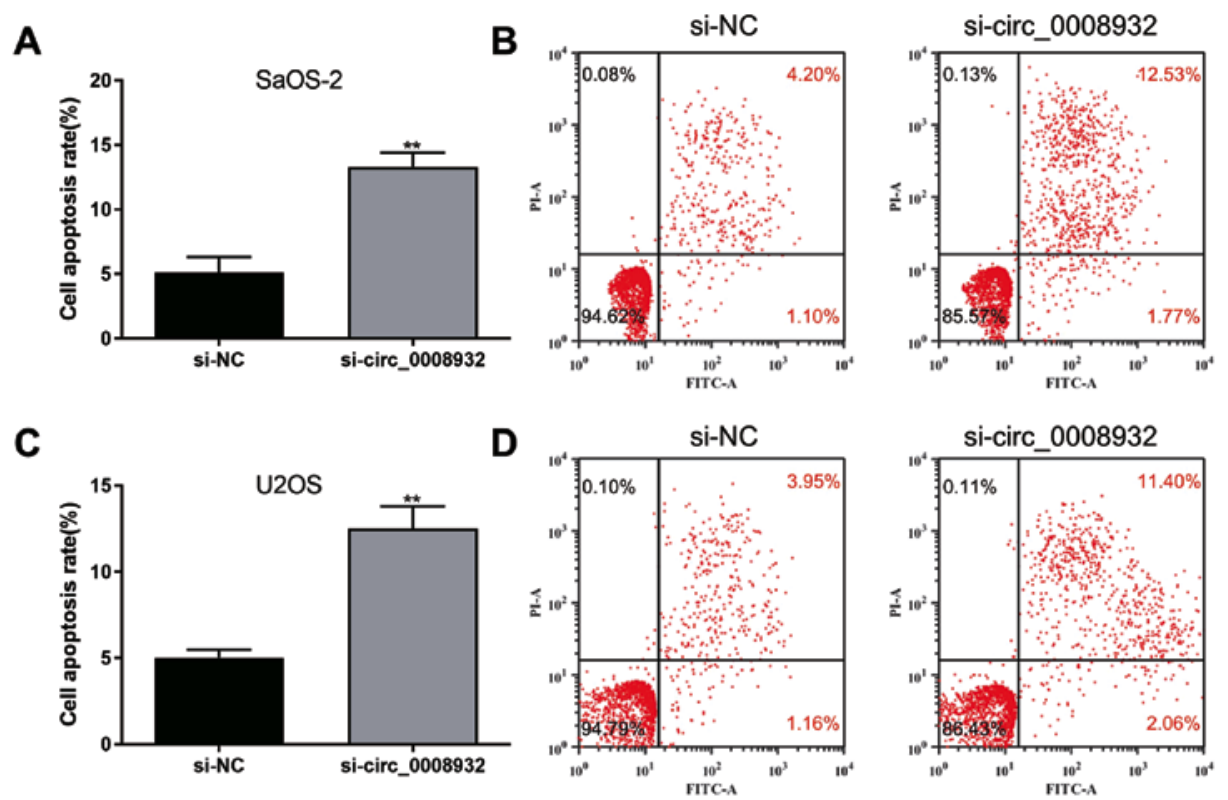

Figure 3. Si-circ_0008932 promotes apoptosis of osteosarcoma cells. (A) Apoptotic rate and (B) representative flow cytometric PI vs. Annexin V dot plots for SaOS-2 cells transfected with si-NC or si-circ_0008932. (C) Apoptotic rate and (D) representative flow cytometric Annexin V vs. PI dot plots for transfected U2OS cells. ${ }^{* *} \mathrm{P}<0.01$ vs. si-NC. Circ, circular RNA; si, short interfering RNA; NC, negative control; PI, propidium iodide.

A
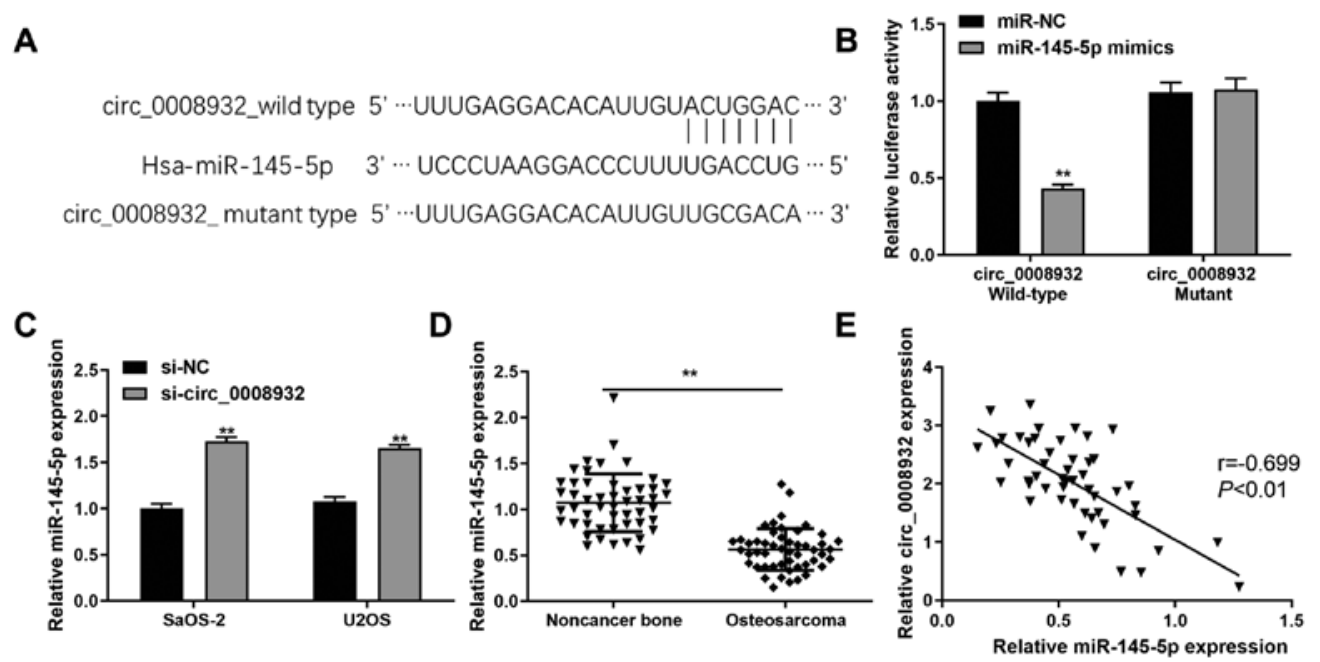

Figure 4. miR-145-5p is a direct target of circ_0008932. (A) Putative binding sequences of circ_0008932 and miR-145-5p. (B) Luciferase vectors containing wild-type or mutant circ_0008932 were co-transfected with miR-NC or miR-145-5p mimics. The luciferase activity was normalized to Renilla. (C) RT-qPCR was used to evaluate the expression of miR-145-5p in SaOS-2 and U2OS cells transfected with si-NC or si-circ_0008932. (D) The expression of miR-145-5p in $50 \mathrm{OS}$ and paired normal tissues was detected by RT-qPCR. (E) Correlation analysis between miR-145-5p and circ_0008932 expression in OS tissues $(\mathrm{r}=-0.699 ; \mathrm{P}<0.01) .{ }^{* *} \mathrm{P}<0.01$ vs. NC. RT-qPCR, reverse transcription-quantitative PCR; circ, circular RNA; miRNA, microRNA; NC, negative control; si, short interfering RNA; hsa, Homo sapiens; OS, osteosarcoma.

increased the expression of miR-145-5p (Fig. 4C). To evaluate the interaction between circ_0008932 and miR-145-5p, their expression levels were determined in OS and paired adjacent normal tissues. The results indicated that the expression of miR-145-5p was significantly decreased in OS tissues (Fig. 4D). Furthermore, the expression of miR-145-5p was inversely correlated with the levels of circ_0008932 (Fig. 4E). Taken together, circ_0008932 was indicated to downregulate miR-145-5p through direct binding, consequently affecting tumor progression in OS.

Si-circ_0008932 inhibits OS cell growth and motility by targeting miR-145-5p. To investigate whether circ_0008932 regulates the proliferation, invasion and migration of OS cells via targeting miR-145-5p, further experiments were performed (Fig. 5). OS cells were transfected with si-NC and si-circ_0008932, or co-transfected with si-circ_0008932, miR-145-5p mimics or inhibitors. The transfection efficiencies of miR-145-5p inhibitors and mimics were confirmed compared with their respective negative controls in both of the OS cell lines (Fig. 5A and B). The results revealed that the proliferation, invasion and migration of OS cells were significantly inhibited, while cell apoptosis was promoted after transfection with si-circ_0008932, and these effects were abolished by miR-145-5p inhibitors (Figs. 5C, E, F, I, S1A and S2A). 

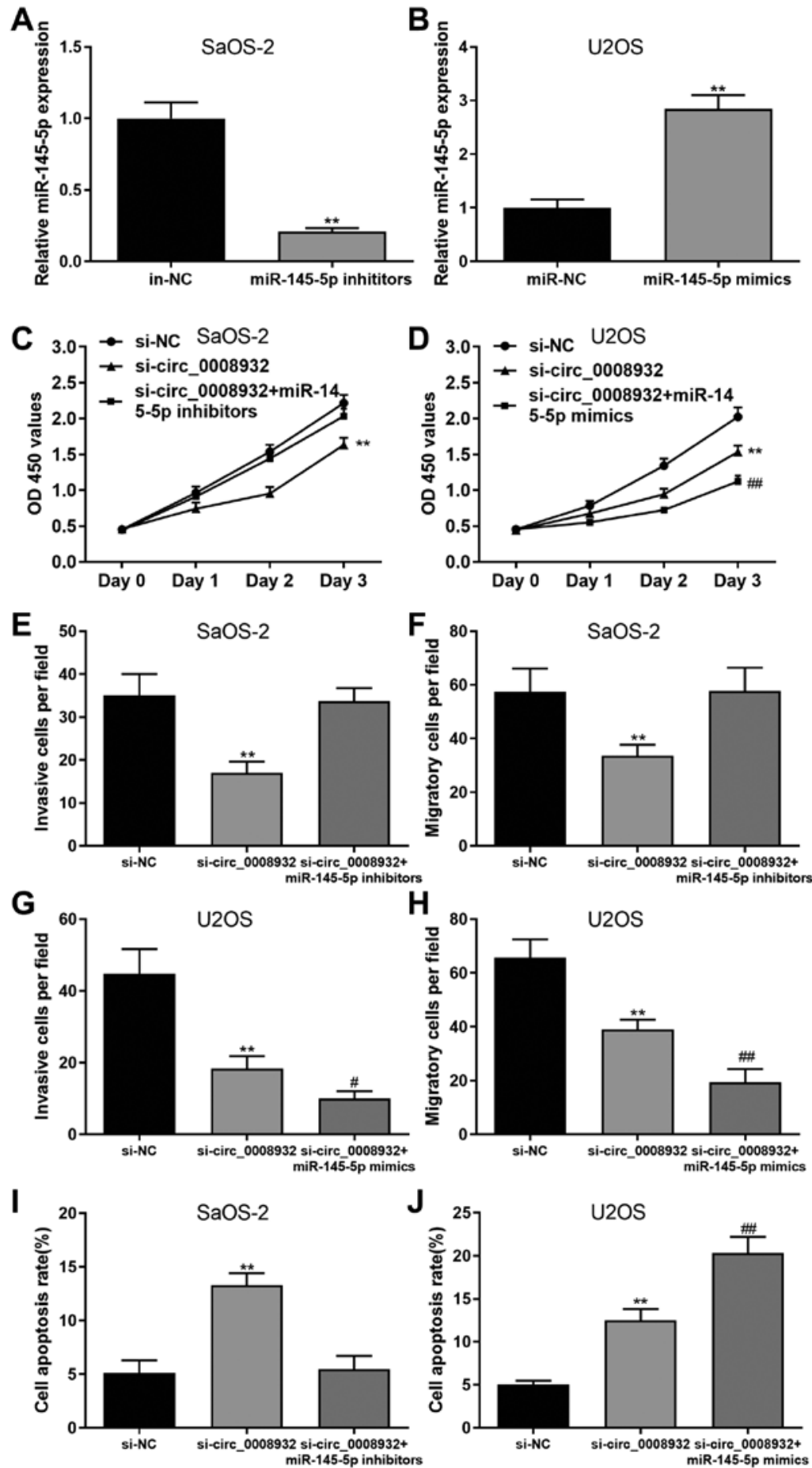

Figure 5. si-circ_0008932 inhibits osteosarcoma cell growth and motility by targeting miR-145-5p. Transfection efficiencies of (A) miR-145-5p inhibitors in SaOS-2 cells and (B) miR-145-5p mimics in U2OS cells were confirmed. A Cell Counting Kit-8 assay was used to determine the proliferation of (C) SaOS-2 co-transfeced with si-circ_0008932 and miR-145-5p inhibitors and (D) U2OS cells co-transfeced with si-circ_0008932 and miR-145-5p mimics. (E and F) Transwell assays were used to examine the (E) invasion and (F) migration of SaOS-2 cells after co-transfection with si-circ_0008932 and miR-145-5p inhibitors. (G and H) Transwell assays were used to determine the $(\mathrm{G})$ invasion and $(\mathrm{H})$ migration of U2OS cells co-transfected with si-circ_0008932 and miR-145-5p mimics. (I and J) Apoptosis was measured in (I) SaOS-2 after co-transfection with si-circ_0008932 and miR-145-5p inhibitors and (J) U2OS cells after co-transfection with si-circ_0008932 and miR-145-5p mimics. ${ }^{* *} \mathrm{P}<0.01$ vs. negative control. ${ }^{*} \mathrm{P}<0.05,{ }^{\# \prime} \mathrm{P}<0.01$ vs. si-circ_0008932. Circ, circular RNA; miRNA, microRNA; NC, negative control; si, short interfering RNA; OD, optical density.

Furthermore, the changes in the biological behaviour of OS cells caused by si-circ_0008932 were enhanced by co-transfection with miR-145-5p mimics (Figs. 5D, G, H, J, S1B and S2B). In summary, silencing of circ_0008932 suppressed the proliferation/motility and promoted apoptosis of OS cells by targeting miR-145-5p.

\section{Discussion}

CircRNA is a type of non-coding RNA widely distributed in eukaryotes. CircRNA is highly stable as it is resistant to exonuclease- or ribonuclease-mediated degradation (13). It has been reported that circRNA is a key regulator of tumor gene expression 
and serves key roles in the signaling networks in oral squamous cell carcinoma, including the Wnt/ $\beta$-catenin and PI3K/AKT pathways (14). Furthermore, upregulation of circ_0008932 was detected in ESCC and knockdown of circ_0008932 suppressed the proliferation, migration and invasion of ESCC cells via the miR-182/Myc axis (9). In addition, circ_0008932 inhibited the migration, proliferation and differentiation of VSMCs through targeting miR-145 (10). However, the detailed functions of circ_0008932 in OS have remained to be fully elucidated.

The results of the present study revealed that circ_0008932 was increased in tumor tissues of patients with OS. In addition, upregulation of circ_0008932 was associated with metastasis in patients with OS. Furthermore, the survival analysis demonstrated that patients with OS with high circ_0008932 expression exhibited poor overall survival. In addition, transfection with si-circ_0008932 inhibited the proliferation, invasion and migration of OS cells, while cell apoptosis was promoted, suggesting that circ_0008932 affected the biological behavior of OS cells. Furthermore, the expression of circ_0008932 was significantly increased in OS cell lines, including HOS, MG-63, SaOS-2 and U2OS.

Previous studies indicated that circRNA acts as an miRNA sponge through competitive binding, consequently leading to loss of function of target miRNA and affect gene expression at the post-transcriptional level (15-19). In the present study, the results of the luciferase reporter assay revealed the interaction between circ_0008932 and miR-145-5p. Furthermore, a negative correlation between the expression of circ_0008932 and miR-145-5p in OS tissues was observed, suggesting that the circ_0008932 and miR-145-5p axis may be involved in the pathogenesis of OS.

Recent studies have revealed the dysregulation of miRNAs in numerous types of cancer, including OS, which is closely associated with tumor cell proliferation, differentiation, apoptosis, autophagy, metastasis and invasion $(20,21)$. Emerging evidence has revealed the involvement of miRNA in the growth and metastasis of OS cells, such as miR-32, miR-142-3p, miR-194, miR-202, miR-217 and let-7a. Furthermore, miR-145-5p was downregulated in OS, which was associated with the metastasis and prognosis of patients with OS $(22,23)$. The regulatory roles of $\mathrm{miR}-145-5 \mathrm{p}$ in the occurrence and development of OS have also been confirmed (24). In addition, Feng et al (25) revealed that miR-145-5p suppressed colorectal cancer cell proliferation, invasion and migration by targeting Fascin-1. Furthermore, Tang et al (26) suggested that the downregulation of miR-145-5p is associated with tumor aggressiveness/metastasis of OS and may be an independent prognostic marker for patients with OS.

In consistency with these results, the present data revealed the downregulation of miR-145-5p in OS specimens, where the expression of miR-145-5p and circ_0008932 were inversely correlated. Furthermore, the present study also indicated that miR-145-5p inhibitors abolished the inhibitory effects caused by si-circ_0008932 on OS progression, whereas miR-145-5p mimics enhanced the changes in biological behavior induced by si-circ_0008932 in OS cells. These results suggested that miR-145-5p is involved in circ_0008932-modulated OS cell proliferation, invasion, migration and apoptosis. However, there were certain limitations to this study. For instance, small RNAs in the samples could have been enriched using a commercial kit prior to the experiments and both SaOS-2 and U2OS cells should be transfected with miR-145-5p mimics and miR-145 inhibitors.

In conclusion, the present results indicated that circ_0008932 may be an oncogenic factor in OS and the inhibitory effects of si-circ_0008932 on the growth and motility of OS cells was through miR-145-5p. To the best of our knowledge, the present study is the first to suggest that the circ_0008932/miR-145-5p axis regulates the proliferation, migration, invasion and apoptosis of OS cells. Of note, this novel signaling pathway of circ_0008932 may be a putative target in future therapies for patients with OS.

\section{Acknowledgements}

Not applicable.

\section{Funding}

No funding was received.

\section{Availability of data and materials}

The datasets used and/or analyzed during the present study are available from the corresponding author on reasonable request.

\section{Authors' contributions}

XS designed the study. CC and XS performed the experiments and analyzed the data. Both authors checked and approved the authenticity of the data. Both authors read and approved the final version of the manuscript.

\section{Ethics approval and consent to participate}

The present study was approved by the Ethics Committee of Chongqing University Cancer Hospital (Chongqing, China). Written informed consent was obtained from all patients for the use of their clinical tissues.

\section{Patient consent for publication}

Not applicable.

\section{Competing interests}

The authors declare that they have no competing interests.

\section{References}

1. Benjamin RS: Adjuvant and neoadjuvant chemotherapy for osteosarcoma: A historical perspective. Adv Exp Med Biol 1257: 1-10, 2020.

2. Garcia MB, Ness KK and Schadler KL: Exercise and physical activity in patients with osteosarcoma and survivors. Adv Exp Med Biol 1257: 193-207, 2020.

3. Anderson PM: Radiopharmaceuticals for treatment of osteosarcoma. Adv Exp Med Biol 1257: 45-53, 2020.

4. Kiany S, Harrison D and Gordon N: The histone deacetylase inhibitor Entinostat/Syndax 275 in osteosarcoma. Adv Exp Med Biol 1257: 75-83, 2020.

5. Li Z, Liu S, Li X, Zhao W, Li J and Xu Y: Circular RNA in Schizophrenia and depression. Front psychiatry 11: 392, 2020. 
6. Meng L, Ding P, Liu S, Li Z, Sang M and Shan B: The emerging prospects of circular RNA in tumor immunity. Ann Transl Med 8: 1091, 2020.

7. Jiang $X$ and Chen D: Circular RNA hsa_circ_0000658 inhibits osteosarcoma cell proliferation and migration via the miR-1227/IRF2 axis. J Cell Mol Med 25: 510-520, 2021.

8. Gu R, Li X, Yan X, Feng Z and Hu A: Circular RNA circ_0032462 enhances osteosarcoma cell progression by promoting KIF3B expression. Technol Cancer Res Treat: Nov 6, 2020 (Epub ahead of print). doi: 10.1177/1533033820943217.

9. Wang J, Zhu W, Tao G and Wang W: Circular RNA circ-LRP6 facilitates Myc-driven tumorigenesis in esophageal squamous cell cancer. Bioengineered 11: 932-938, 2020.

10. Hall IF, Climent M, Quintavalle M, Farina FM, Schorn T, Zani S, Carullo P, Kunderfranco P, Civilini E, Condorelli G and Elia L: Circ_Lrp6, a Circular RNA enriched in vascular smooth muscle cells, acts as a sponge regulating miRNA-145 function. Circ Res 124: 498-510, 2019.

11. Prats AC, David F, Diallo LH, Roussel E, Tatin F, Garmy-Susini B and Lacazette E: Circular RNA, the key for translation. Int J Mol Sci 21: 8591, 2020.

12. Livak KJ and Schmittgen TD: Analysis of relative gene expression data using real-time quantitative PCR and the 2(-Delta Delta C(T)) method. Methods 25: 402-408, 2011.

13. Tsitsipatis D and Gorospe M: Practical guide forcircular RNA analysis: Steps, tips, and resources. Wiley Interdiscip Rev RNA 12: e1633, 2021.

14. Saikishore R, Velmurugan P, Ranjithkumar D, Latha R, Sathiamoorthi T, Arun A, Ravi AV and Sivakumar S: The circular RNA-miRNA axis: A special RNA signature regulatory transcriptome as a potential biomarker for OSCC. Mol Ther Nucleic Acids 22: 352-361, 2020

15. Wen J, Liao J, Liang J, Chen X, Zhang B and Chu L: Circular RNA HIPK3: A key circular RNA in a variety of human cancers. Front Oncol 10: 773, 2020.

16. $\mathrm{Su} \mathrm{Q}$ and $\mathrm{Lv} \mathrm{X}$ : Revealing new landscape of cardiovascular disease through circular RNA-miRNA-mRNA ax is Genomics 112: 1680-1685, 2020.

17. Gong C, Zhou X, Lai S, Wang L, Liu J, Yanming X and Xu Y: Long noncoding RNA/Circular RNA-miRNA-mRNA axes in Ischemia-Reperfusion injury. Biomed Res Int 2020: 8838524, 2020.
18. Wong RR, Abd-Aziz N, Affendi S and Poh CL: Role of microRNAs in antiviral responses to dengue infection. J Biomed Sci 27: 4, 2020.

19. Boca S, Gulei D, Zimta AA, Onaciu A, Magdo L, Tigu AB, Ionescu C, Irimie A, Buiga R and Berindan-Neagoe I: Nanoscale delivery systems for microRNAs in cancer therapy. Cell Mol Life Sci 77: 1059-1086, 2020.

20. Ebrahimi N, Aslani S, Babaie F, Hemmatzadeh M, Pourmoghadam Z, Azizi G, Jadidi-Niaragh F and Mohammadi H: MicroRNAs implications in the onset, diagnosis, and prognosis of osteosarcoma. Curr Mol Med: Dec 3, 2020 (Epub ahead of print).

21. Fu Y, Wang Y, Bi K, Yang L, Sun Y, Li B, Liu Z, Zhang F, Li Y, Feng C and Bi Z: MicroRNA-208a-3p promotes osteosarcoma progression via targeting PTEN. Exp Ther Med 20: 255, 2020.

22. Kushlinskii NE, Fridman MV and Braga EA: Molecular mechanisms and microRNAs in osteosarcoma pathogenesis. Biochemistry (Mosc) 81: 315-328, 2016.

23. Zhou Y, Feng D, Gu X, Gao A and Liu Y: The role and clinical significance of long noncoding RNA zinc finger E-box-binding homeobox two antisense RNA 1 in promoting osteosarcoma cancer cell proliferation, inhibiting apoptosis and increasing migration by regulating miR-145. Anticancer Drugs 32: 168-177, 2021.

24. Li X, Liu Y, Zhang X, Shen J, Xu R, Liu Y and Yu X: Circular RNA hsa_circ_0000073 contributes to osteosarcoma cell proliferation, migration, invasion and methotrexate resistance by sponging miR-145-5p and miR-151-3p and upregulating NRAS. Aging (Albany NY) 12: 14157-14173, 2020.

25. Feng Y, Zhu J, Ou C, Deng Z, Chen M, Huang W and Li L: MicroRNA-145 inhibits tumour growth and metastasis in colorectal cancer by targeting fascin-1. Brit J Cancer 110: 2300-2309, 2014

26. Tang M, Lin L, Cai H, Tang J and Zhou Z: MicroRNA-145 downregulation associates with advanced tumor progression and poor prognosis in patients suffering osteosarcoma. Onco Targets Ther 6: 833-838 2013.

This work is licensed under a Creative Commons Attribution-NonCommercial-NoDerivatives 4.0 International (CC BY-NC-ND 4.0) License. 